\title{
Targeted DNA methylation represses two enhancers of FLOWERING LOCUS T in Arabidopsis thaliana
}

\author{
Johan Zicola', Liangyu Liu², Petra Tänzler and Franziska Turck ${ }^{1 *}{ }^{1 *}$
}

FLOWERING LOCUS T (FT) plays a major role in regulating the floral transition in response to an inductive long day photoperiod in Arabidopsis thaliana. Expression of FT in leaves is dependent on the distal transcriptional enhancer Block C, located 5-kilobases (kb) upstream of the transcriptional start site (TSS). We expressed an inverted repeat of Block C to induce local DNA methylation and heterochromatin formation, which lead to FT downregulation in an inductive photoperiod. Using targeted DNA methylation as a tool to uncover further regulatory regions at the FT locus, we identified Block E, located $1 \mathrm{~kb}$ downstream of the gene, as a novel enhancer of FT. As Block $C, B l o c k E$ is conserved across Brassicaceae and located in accessible chromatin. Block $C$ and $E$ act as additive transcriptional enhancers that, in combination with the proximal $F T$ promoter, control expression of $F T$ in response to photoperiod in the leaf phloem.

T he distal transcriptional enhancer of FT, Block $C$, contains several consensus transcription factor binding sites (TFBS $)^{1}$ including a conserved CCAAT box required for full promoter activity $^{2}$. The CCAAT box motif acts as binding site for trimeric nuclear transcription factor-Y (NF-Y) complexes ${ }^{2,3}$. CONSTANS is the key transcription factor responsible for inducing expression of $F T$ in long days ${ }^{4}$. CONSTANS interacts with NF-YB and NF-YC subunits to form a trimeric complex ${ }^{5,6}$, which binds with high affinity to CCACA elements ${ }^{3}$.

Transcriptional enhancers (hereafter, enhancers) are binding platforms for multiple transcription factors. Enhancers were shown to physically interact with the promoters of their target genes where they participate in recruiting the transcriptional machinery ${ }^{7}$. So far, relatively few enhancers were characterized in plants compared to their counterparts in animals ${ }^{8}$. Two studies applied chromosomal conformation capture (3C) to measure interactions between regulatory regions at the $F T$ locus ${ }^{2,9}$. Both studies detected a weak interaction between the TSS and Block $C$ and stronger interactions between the TSS and regions located in the interspace between Block $C$ and the TSS; however, the peak interacting regions differed between the studies. Thus, the contribution of Block $C$ and other regulatory regions to $F T$ expression in the native chromatin context requires further analysis.

In plants, de novo DNA methylation is established by the RNAdependent DNA methylation (RdDM) pathway ${ }^{10}$. The possibility to target DNA methylation to specific regions through the expression of inverted repeats (IR), which generate double-stranded RNA that can be directly processed into the RdDM pathway, provides a tool to alter gene activity by transcriptional gene silencing (TGS) ${ }^{11}$. In Arabidopsis, IR-mediated DNA methylation of the promoters of TOO MANY MOUTHS (TMM) and FT induced TGS ${ }^{12,13}$. Here we demonstrate that IR-mediated DNA methylation can repress the activity of distal regulatory regions, allowing us to uncover novel regulatory regions and study their effect on gene expression in the native genomic context.

\section{Results}

IR-induced transgenerational late flowering. Transgenic lines expressing an IR of Block $C$ to target DNA methylation at Block $C$ and assess the effect on FT expression were generated in the Columbia (Col-0) background (referred to as wild type, WT) (Fig. 1a). Two independent single-insertion lines and their non-transgenic siblings were propagated until generation T6 (Fig. 1b). Flowering time was assessed for each generation in strongly $F T$-inductive long day photoperiod (16h light $/ 8 \mathrm{~h}$ dark) and moderately inductive midday conditions ( $12 \mathrm{~h}$ light $/ 12 \mathrm{~h}$ dark). To compare flowering time across generations, four generations (T3 to T6) for each line were grown simultaneously under mid-day conditions. Flowering was significantly delayed compared to wild type for the transgenic lines across generations while non-transgenic sibling lines showed a marginal delay in flowering (Fig. 1c). A similar delay in flowering was observed in several independent experiments conducted in long day conditions (Supplementary Fig. 1).

FT expression was assessed for the four lines at generations T3 and T6 in long day conditions. Corresponding to their respective flowering phenotypes, the transgenic lines no. 15-2 and no. 27-4 but not their non-transgenic siblings no. 15-3 and no. 27-3 showed a statistically significant reduction in FT expression for both generations (Fig. 1e). In conclusion, presence of the IR targeting Block $C$ clearly correlated with delayed flowering in inductive photoperiod and reduced expression of FT. Although the delay in flowering was observed in non-transgenic siblings, no significant changes in FT expression were detected.

DNA methylation and heterochromatin formation is induced at Block C. Previous studies have shown that 24-nucleotides (nt) small RNAs (smRNAs) are required for effective TGS through DNA methylation $^{14,15}$. Expression of an IR generates dsRNA, which can be directly processed by DICER LIKE 3 (DCL3) to integrate the RdDM pathway ${ }^{10}$. We sequenced the smRNAs in transgenic and non-transgenic lines at generation T6 and found smRNAs mapping specifically to the IR-targeted region exclusively in the transgenic

${ }^{1}$ Max Planck Institute for Plant Breeding Research, Cologne, Germany. ${ }^{2}$ College of Life Sciences, Capital Normal University, Beijing, China.

*e-mail: turck@mpipz.mpg.de 
a
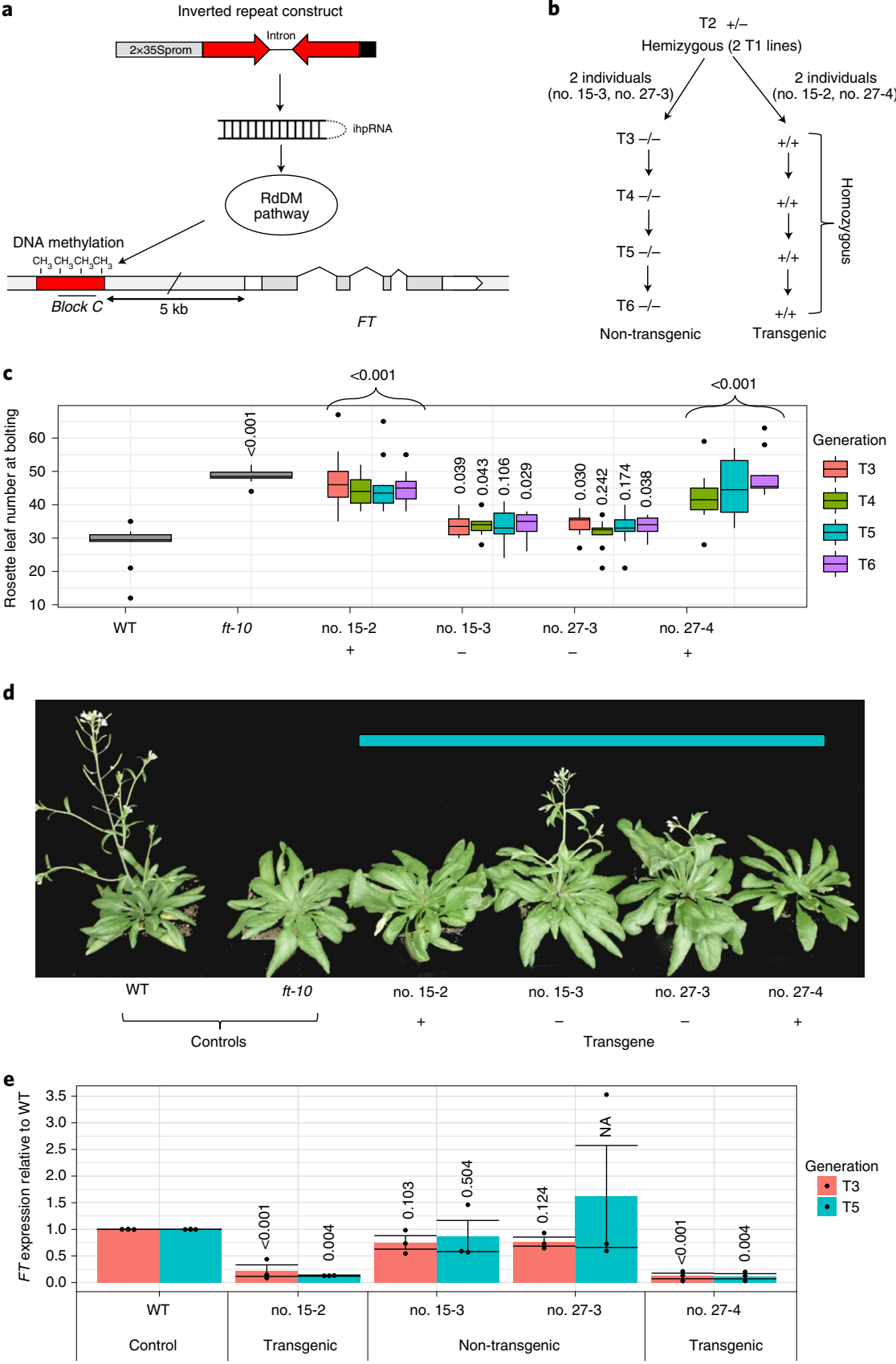

Fig. 1 | Floral transition is delayed and FT expression downregulated in Block C IR-containing plants. a, Cartoon of IR-mediated DNA methylation at Block C. An IR including Block C sequence (red arrows) is driven by a strong Cauliflower Mosaic Virus (CaMV) 35 S promoter with duplicated enhancer (grey box), separated by the intron 1 of WRKY33, and terminated by the CaMV $35 \mathrm{~S}$ terminator (black box). Transcription of the construct forms an intron-containing hairpin RNA (ihpRNA) that can be processed into the RdDM pathway to trigger de novo DNA methylation at the target region (in red) containing Block C (black line), which is located $5 \mathrm{~kb}$ upstream of $F T$. b. Two independent T2 transgenic lines hemizygous for the transgene were propagated and both homozygous transgenic and non-transgenic siblings were selected. These four lines were propagated from T3 to T6 generation.

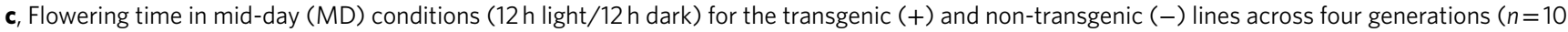
plants per line and generation). Centre lines of box plots show the medians, box limits for the 25th and 75th percentiles, whiskers extend 1.5 times the interquartile range from the 25 th and 75 th percentiles, outliers are represented by dots. The transfer DNA (T-DNA) insertion line ft-10 is a FT loss-offunction allele and was used as late-flowering control. The transgenic line no. 27-4 at T3 is not displayed in c due to bad germination. $\mathbf{d}$, Phenotype of one representative plant $(n=10)$ from wild type (WT) and ft-10 and the four lines at generation T5 from the same experiment (indicated by the cyan box). e, FT expression measured in two-week-old long day (LD) grown seedlings at Zeitgeber time (ZT) 16. FT expression was normalized to the expression of PP2A and the wild type was set to one. The error bars show s.e.m. and black dots indicate the values of the three biological replicates. For $\mathbf{c}$ and $\mathbf{e}$, analysis of variance (ANOVA) followed by post hoc Dunnett's test were performed for each generation (one-sided, wild type as a control group, $P$ values indicated above each bar). Note that for $\mathbf{e}$, values for line no. 27-3 generation T5 were excluded from the statistical analysis due to an extreme value of $F T$ expression for one of the replicates. 
a

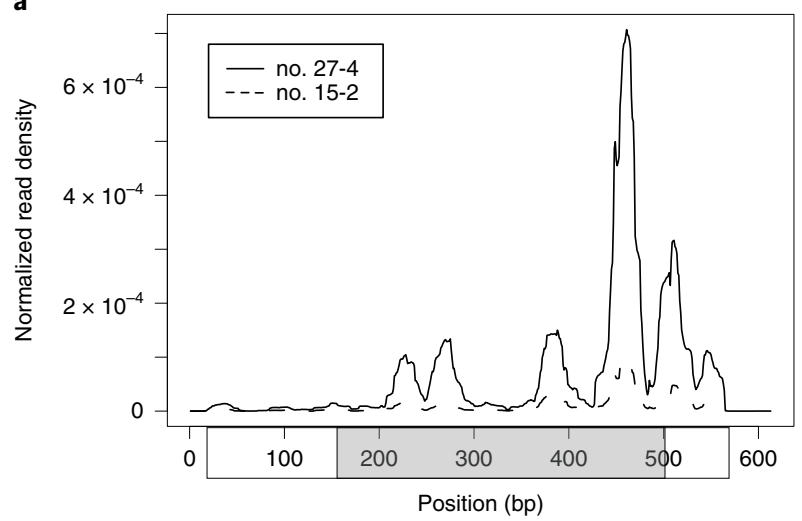

b

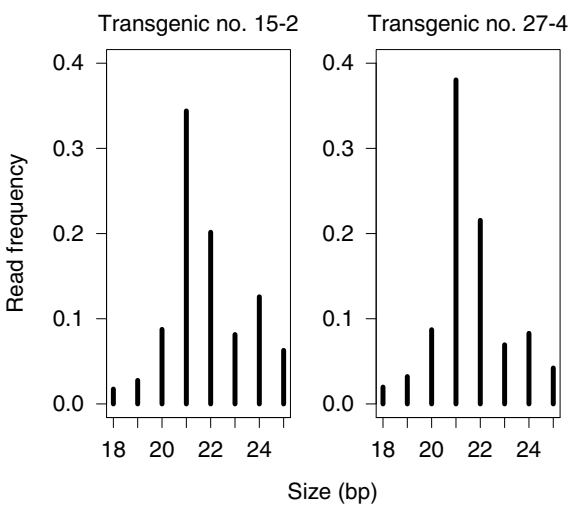

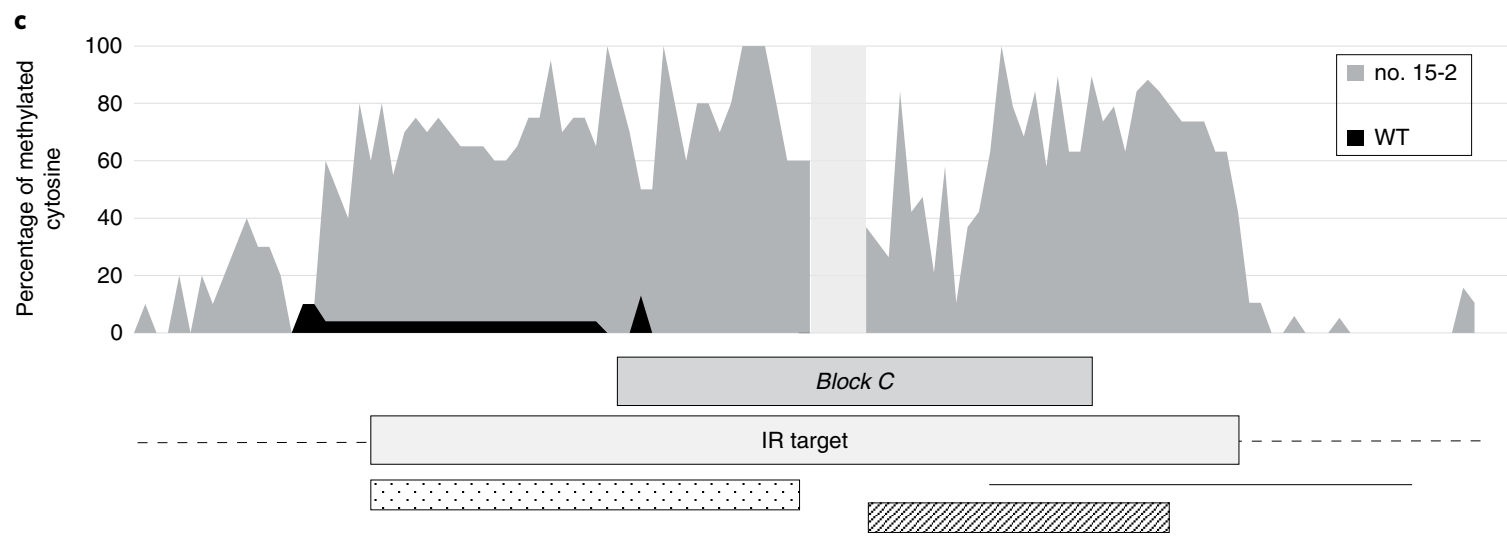

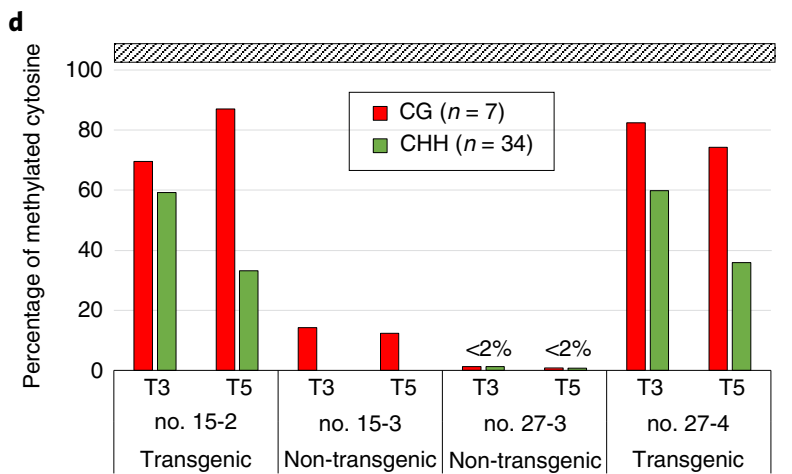

e

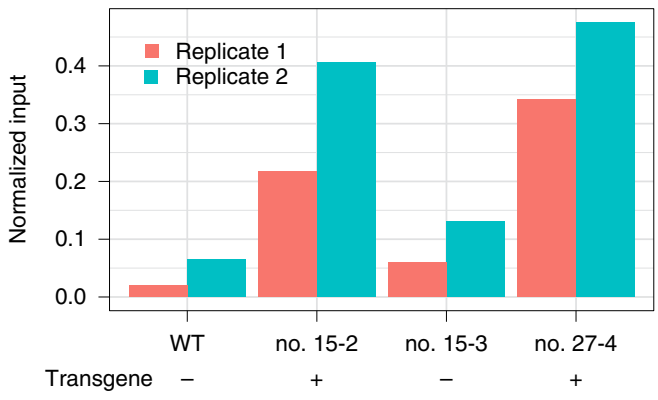

Fig. 2 DNA methylation is set at Block C in IR-containing plants. a, Density of reads mapping to the IR target region for both transgenic lines no. 15-2 and no. 27-4 (generation T6) normalized by the total number of mapped reads. Non-transgenic lines are not shown since no smRNAs could be found. The IR target region (light grey box) encompasses Block C (dark grey box). b. Frequency distribution of read sizes in the IR target region for both transgenic lines no. 15-2 and no. 27-4 (generation T6). c, Average level of DNA methylation at each cytosine position for wild type (WT) (black) and transgenic line no. 15-2 (generation T3) (dark grey) at the IR target region (light grey box), which encompasses Block C (dark grey box). The vertical light grey box represents a region containing four cytosines with unknown methylation status. In total, 116 cytosines were assessed with at least 10 clones per position. The dotted box indicates a region examined for DNA methylation in wild type, transgenic lines nos. 15-2 and 27-4, and non-transgenic sibling no. 15-3 in Supplementary Fig. 2. d Average level of DNA methylation in all contexts at the Block $\mathrm{C}$ region indicated by the box with diagonal hatching in $\mathbf{c}$ for two transgenic lines (nos. 15-2 and 27-4) and their non-transgenic siblings (nos. 15-3 and 27-3) for two different generations (T3 and T5) in CG and CHH contexts ( $\mathrm{CHG}$ context is absent in the sequence), in which ' $\mathrm{C}$ ' indicates methylated cytosine and ' $\mathrm{H}$ ' indicates any nucleotide residue other than guanine (G). At least eight clones were examined. The number of cytosines for each context is indicated. $\mathbf{e}$, An overlapping region of $213 \mathrm{bp}$ between the IR target region and the flanking endogenous region was used as probe (black line in c) to assess the level of H3K9me2 at the endogenous Block C in wild type three lines: transgenic lines nos. 15-2 and 27-4, and non-transgenic line no. 15-3 (generation T5). The percentage of input at Block $C$ was normalized to the percentage of input at the transposable element (cl2-ta22), which is used as $\mathrm{H} 3 \mathrm{~K} 9$ me2-rich region control. The normalized values of two biological replicates are shown.

sibling lines. Thus, presence of the IR was required for smRNA production, which was limited to the inner 400 base pairs $(\mathrm{bp})$ of the IR stem-loop (Fig. 2a). We also found smRNAs mapping at the intron of WRKY33, which is used to separate the inverted repeats in the transgene (data not shown). The predominant smRNA class mapping to the IR target region was 21 -nt long, followed by 22 -nt and 24-nt smRNA classes (Fig. 2b). Transgenic line no. 27-4 generated about seven times more smRNAs than line no. 15-2 but the 
reduction of $F T$ expression was comparable in both lines, indicating that the pathway was saturated for smRNAs (Fig. 2a,b).

Bisulfite conversion was performed to assess DNA methylation level at Block $C$. We confirmed the absence of DNA methylation at Block $C$ in Col-0 wild type seedlings as previously reported ${ }^{16}$ (Fig. 2c). In the transgenic lines, DNA methylation was induced at the IR target region including Block $C$, with levels of DNA methylation varying from 10 to $100 \%$ for each cytosine position (Fig. 2c, d and Supplementary Fig. 2). DNA methylation was also detected at sites flanking the IR target region up to $100 \mathrm{bp}$ up- and downstream, although no smRNAs mapped to these regions (Fig. 2c). To assess long-range spreading of DNA methylation, a 300bp region located between Block $C$ and the FT TSS was selected as control. No DNA methylation was observed in the control region, indicating that DNA methylation did not spread from Block $C$ towards the TSS of FT (Supplementary Fig. 3). We compared DNA methylation levels at generation T3 and T5 and found that CG methylation was maintained in both transgenic lines and to a much lower extent in non-transgenic lines with about $10 \%$ for no. $15-3$ and few percent for no. 27-3 (Fig. 2d). At this reduced level, CG methylation was maintained from $\mathrm{T} 3$ to $\mathrm{T} 5$ generation in absence of the transgene (Fig. 2d). CHH methylation decreased in the transgenic lines with progressing generations while it was near zero in the non-transgenic lines no. 15-3 and no. 27-3 (Fig. 2d). Altogether, these results indicate that, on IR loss, CG methylation is partially maintained and the level of maintenance varies between lines.

Methylated DNA can recruit histone methyltransferase KRYPTONITE (KYP), which di-methylates lysine 9 of histone $\mathrm{H} 3$ (H3K9me2) leading to chromatin compaction ${ }^{17}$. H3K9me2 in turn recruits CHROMOMETHYLASE 2 (CMT2) and CHROMOMETHYLASE 3 (CMT3) promoting DNA methylation in $\mathrm{CHH}$ and $\mathrm{CHG}$ contexts, respectively ${ }^{18}$. We assessed whether IR-induced DNA methylation resulted in H3K9me2 deposition at Block $C$ using chromatin immunoprecipitation (ChIP). In two independent experiments, $\mathrm{H} 3 \mathrm{~K} 9 \mathrm{me} 2$ levels were increased in the transgenic lines compared to wild type while no increase was observed for the non-transgenic sibling no. 15-3 (line no. 27-3 was not tested) (Fig. 2e).

$F T$ is regulated by the downstream enhancer Block $E$. Since IR-targeted DNA methylation at Block $C$ downregulated FT expression, we surveyed other regions potentially involved in $F T$ regulation with this method (Fig. 3a). We designed IRs for the previously identified Block $B$ and a region described as Col-0 insertion, present in around $25 \%$ of $A$. thaliana accessions ${ }^{9}$ (Fig. 3a). These regions correspond to two distinct regions shown to interact with the proximal FT promoter based on $3 \mathrm{C}$ data $^{2,9}$. DNA methylation was not previously reported at Block $B$, while the Col-0 insertion was shown to carry methylation ${ }^{16}$. Our data from Col-0 did not reveal DNA methylation in the control region adjacent to the $3^{\prime}$ end of Block $B$ (Supplementary Fig. 3) and confirmed the presence of DNA methylation at the Col-0 insertion region (Supplementary Fig. 4). Expression of IRs induced comparable DNA methylation at all respective target regions leading to an effective increase in $\mathrm{CHG}$ and $\mathrm{CHH}$ at the Col-0 insertion and in all contexts at Block B (Fig. 3b). Although no statistically significant differences in FT expression were detected for plants expressing the IR targeting Col-0 insertion region (Fig. 3c), a mild but significant late-flowering phenotype was detected (Fig. 3d).

We extended the previous phylogenetic analysis that had identified Block $C$ by adding more orthologous sequences from Brassicaceae species that have become available on the Phytozome database $^{19}$. A region of $600 \mathrm{bp}$ located $1 \mathrm{~kb}$ downstream of $F T$, named hereafter Block E, was highly conserved among all available sequences (Fig. 3a). Besides phylogenetic conservation, both, Block $E$ and Block $\mathrm{C}$, are located in highly accessible chromatin ${ }^{20}$
(Fig. 3a). Further analysis revealed that Block $C$ and $E$ share several super-conserved shadows that overlap with potential TFBSs, such as an I-box, a RE-alpha box and two CCAAT boxes (Supplementary Fig. 5a,b). Furthermore, Block $E$ contains a G-box (CACGTG), which is located in the binding peak of PHYTOCHROME INTERACTING FACTOR 4 (PIF4) found in ChIP-seq data ${ }^{21}$ (Supplementary Fig. 5a).

We designed an IR to target Block $E$ and test whether DNA methylation deposition would, as for Block $C$, affect flowering time (Fig. 3a). Expression of Block E IR statistically significantly delayed flowering time compared to the wild type (Fig. 3e). Consistent with the delay in flowering time, FT expression was reduced in transgenic lines expressing an IR directed against Block $E$ (Fig. 3f), which correlated with increased DNA methylation in all sequence contexts (Fig. 3b) and presence of 21-24 smRNAs mapping specifically at the target sequence (Supplementary Fig. 6a-c).

To study the genetic interaction between the two distal regulatory regions, we assessed the flowering time of F1 crosses between Block C and Block E IRs. F1 plants hemizygous for both IRs flowered significantly later than their parents and also later than control F1 crosses to Col-0, which were hemizygous for only one IR. Taken together, the two regulatory regions play an additive role in regulating flowering time (Fig. $3 g$ and Supplementary Fig. 7).

Block $E$ and $C$ enhance the photoperiod response encoded at the FT promoter. Considering the number of shared TFBSs between Block $E$ and $C$, we tested the cis-regulatory function of Block $E$ in stably transformed reporter gene plant lines. We have previously shown that Block $C$ fused to Block $A$, but not Block $A$ alone, can drive GUS expression in leaves ${ }^{9}$. Block $E$ was fused to the minimal promoters of FT (Block A) or NOS (minNOSp) in both forward and reverse orientations. While Block $C$ was shown to drive GUS expression only when fused to Block $A$, Block $E$ was able to drive GUS expression with both minimal promoters (Fig. 4a) ${ }^{9}$. In combination with Block A, Block $E$ activity was orientation-dependent: Block $E$ in reverse orientation could drive GUS expression while Block $E$ in sense orientation could not. This was consistently observed for the seven single-insertion lines obtained for each construct (Fig. 4a and Supplementary Fig. 8). However, this orientation-dependent activity was not visible when $\operatorname{minNOSp}$ was used as minimal promoter: five out of nine single-insertion lines showed GUS expression with Block $E$ in sense orientation and all five lines with Block $E$ in reverse orientation (Fig. 4a and Supplementary Fig. 8). In most cases, GUS signal was found in the vasculature of the leaves, where FT is expressed, but some lines also showed a GUS signal in the roots or shoot apical regions (Supplementary Fig. 8).

To assess if Block $E$ regulated expression in the response to photoperiod, we compared reporter gene expression between seedlings grown either continuously for 3 weeks in a short day or after a 2 day shift to a long day. We used only constructs of Block $E$ in reverse orientation as these gave the strongest signal (Fig. $4 \mathrm{a}$ and Supplementary Fig. 8). GUS transcript analysis by quantitative PCR with reverse transcription (qRT-PCR) of samples collected at ZT16 showed a clear induction in long day conditions for reporter constructs containing either Block $C$ or Block $E$ fused to Block $A$, while fusions to $\operatorname{minNOS}$ were not responsive (Fig. 4b).

\section{Discussion}

We showed that IR-mediated DNA methylation of Block $C$ lead to the downregulation of FT expression and was associated with late flowering and $\mathrm{H} 3 \mathrm{~K} 9 \mathrm{me} 2$ deposition (Figs. 1 and 2). We used the same rational to characterize other putative cis-regulatory elements of FT. We found that IRs targeting the previously defined Block $B$ and the Col-0 insertion regions had a mild but statistically significant effect on flowering while an IR targeting the novel element Block $E$ strongly delayed flowering (Fig. 3e,g). Block $E$ is, 

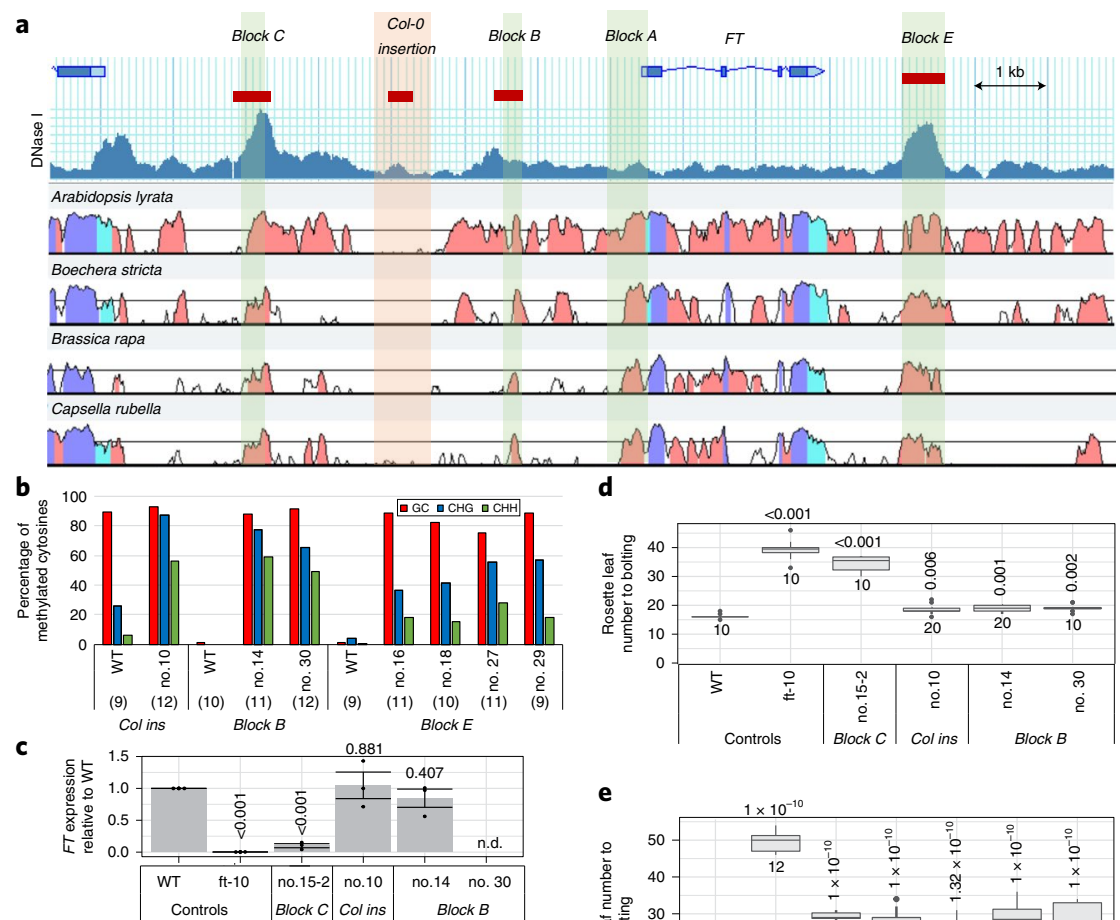

f

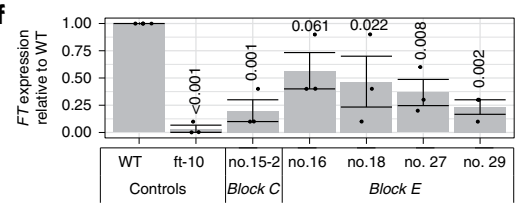

e
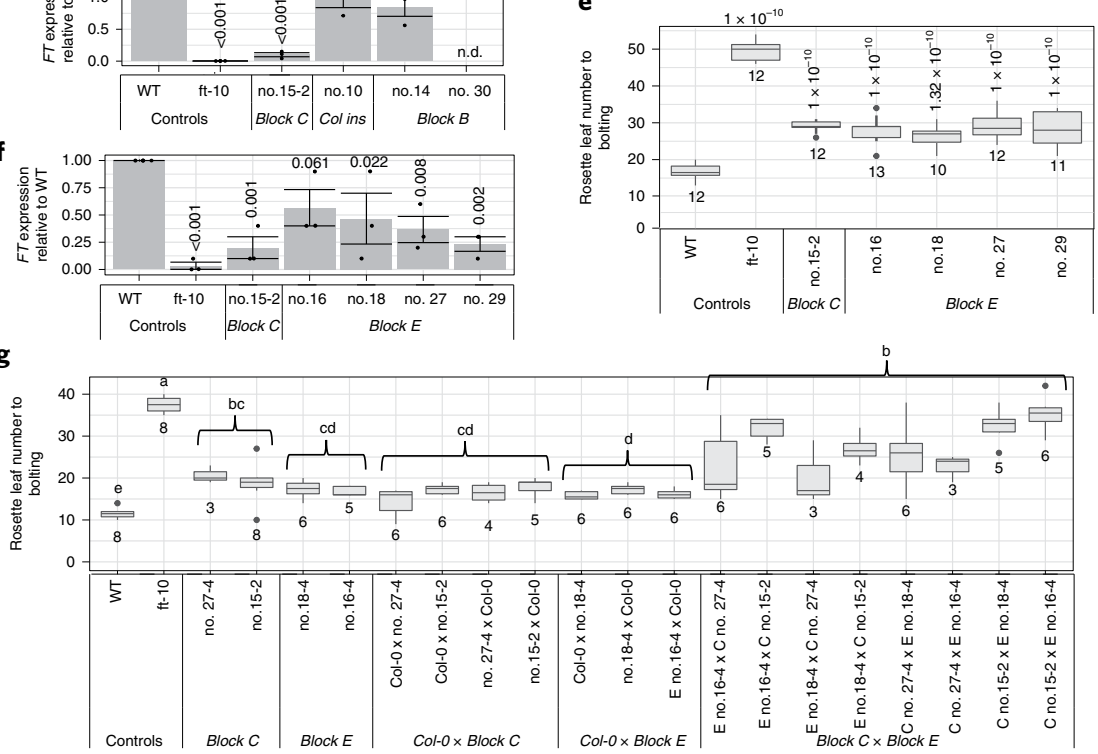

Fig. 3 | Block $E$ is a novel regulatory element located downstream of FT. a, Chromatin accessibility and conservation level at the $F T$ locus. Chromatin accessibility from published DNase-seq data (labelled DNase I) ${ }^{20}$. Phylogenetic shadowing showing the level of sequence homology between $A$. thaliana and four related Brassicaceae species. Red, cyan and purple colours code indicate intergenic regions and introns, Untranslated Regions (UTRs) and exons, respectively. Green shadows indicate the previously defined Block $C$ and Block $B$, and the newly defined Block $E$. The orange shadow indicates the location of the Col-O insertion. Red filled boxes indicate regions targeted by IRs. $\mathbf{b}$, Average DNA methylation level assessed at the different regions targeted with IR and by methylation context. The number of analysed clones is indicated in parentheses. c, Expression level of $F T$ compared to wild type (WT) for the different transgenic lines containing IRs. Error bars show s.e.m. and black dots indicate the values of the three biological replicates. Col-0 and ft-10 were used as control for wild type and null-allele flowering time phenotype, respectively. FT expression was normalized to PP2A and the wild type value was set to one. The seeds of the transgenic line no. 30 germinated poorly and did not provide comparable FT expression data (n.d., no data). ANOVA followed by post hoc Dunnett's test was performed (one-sided, wild type as control group, $P$ values indicated above each bar). $\mathbf{d}$, Flowering time in long day (LD) as number of rosette leaves at bolting for wild type, $\mathrm{ft}-10$ and IR lines as indicated. Centre lines of box plots show the medians, box limits indicate the 25 th and 75th percentiles, whiskers extend 1.5 times the interquartile range from the 25th and 75th percentiles, outliers are represented by dots. Number of phenotyped plants indicated under each box plot. Significance tested by ANOVA followed by post hoc Dunnett's test (one-sided, wild type as control group, $P$ values indicated above each bar). e, Flowering time in long day number of rosette leaves at bolting for wild type, ft-10 and IR lines as indicated. Box plots and statistical analysis as described for $\mathbf{d}$. f, Expression level of $F T$ compared to wild type for the different transgenic lines containing IRs. Error bars show s.e.m. and black dots indicate the values of the three biological replicates. ANOVA followed by post hoc Dunnett's test was performed (one-sided, wild type as control group, $P$ values indicated above each bar). $\mathbf{g}$, Flowering time in long day for two independent Block C IR and Block $E$ IR lines (generation T3) and their reciprocal F1 crosses, F1 crosses to (wild type) Col-0, Col-0 and ft-10 used as controls. Box plots as described for $\mathbf{d}$. Statistical analysis by Kruskall-Wallis multiple-comparison test to rank differences between wild type, ft-10 and the groups indicated by curly brackets, which correspond to pooled data for each construct and F1 cross. Groups sharing the same letters are not significantly different alpha risk $=5 \%$ with Bonferroni correction). Vertical labels for F1 crosses indicate direction of the cross (mother $\times$ father). 

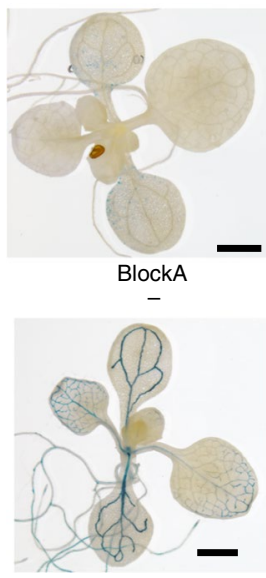

BlockErev:BlockA

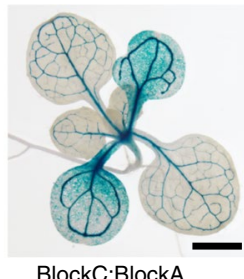

BlockC:BlockA

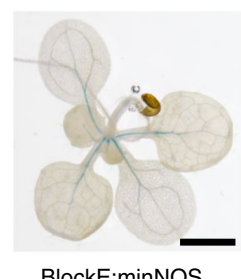

BlockE:minNOS

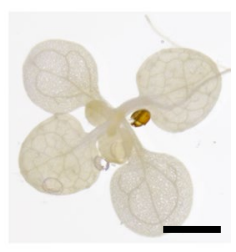

BlockE:BlockA

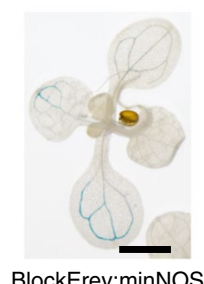

b

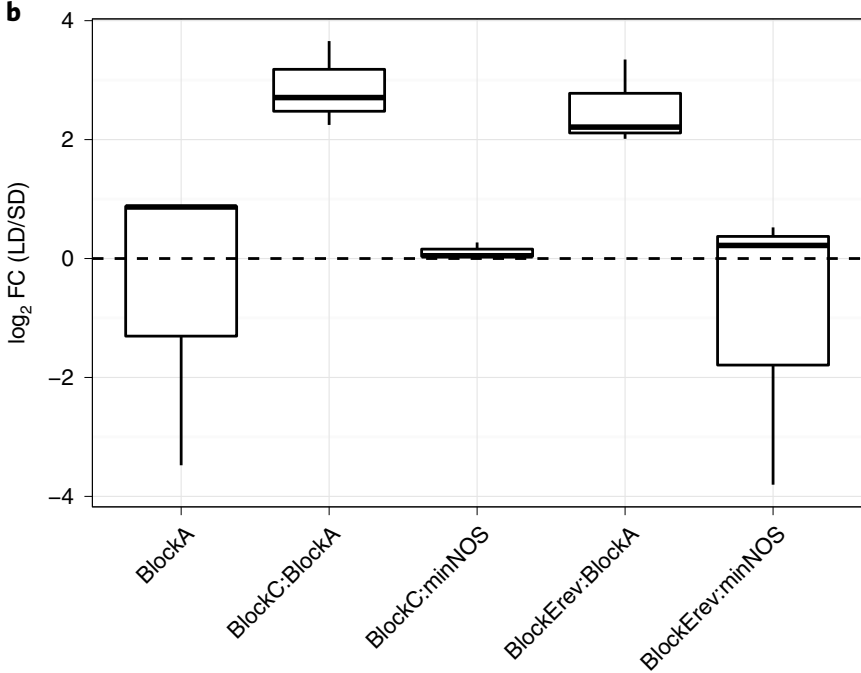

Fig. 4 | Block E drives GUS expression in long day conditions. a, GUS staining of 2-week-old seedlings containing GUS reporter constructs with the following promoters: BlockA:GUS (negative control, two independent lines tested with similar results), BlockC:BlockA (positive control, two independent lines tested with similar results), BlockE:Block $A$ (seven independent lines tested with similar results), BlockErev:BlockA (Block $E$ in reverse orientation, seven independent lines tested with similar results), BlockE:minNOSp (nine independent lines tested with similar results) and BlockErev:minNOSp (five independent lines tested with similar results) fused to the GUS reporter gene. The plants were grown in long day (LD) conditions. Scale bars, $1 \mathrm{~mm}$. b, GUS transcript levels in 3-week-old seedlings containing the constructs as in a with the addition BlockC:minNOS construct. Centre lines of box plots show the medians, box limits indicate the 25th and 75th percentiles, and whiskers extend 1.5 times the interquartile range from the 25 th and 75 th percentiles. All plants were grown in two replicates in short day (SD) conditions then split in long day and short day conditions for 2 days. Samples were collected at ZT16. Expression of GUS was normalized to PP2A reference gene and the ratio of expression between long and short day conditions was calculated as $\log _{2}$ FC (LD/SD). Three independent lines per construct were considered as a biological replicates.

as Block $C$, highly conserved across Brassicaceae, contains similar putative TFBSs and is located in accessible chromatin. We report here a downregulation of a gene by targeting DNA methylation at distal enhancers in $A$. thaliana.

IRs were previously used to completely silence gene expression of TMM and FT by targeting promoter regions ${ }^{12,13}$. In comparison,

our IR targeting distal enhancer regions lead to partial downregulation of FT. This discrepancy can be due to the more general regulatory role of promoters compared to enhancers, which leads to a more deleterious effect of DNA methylation and modification of the chromatin structure from the former. In addition, IR promoter targeting can induce secondary short interfering RNAs (siRNAs) from the coding region to be generated and subsequently trigger post-transcriptional gene silencing 22,23 . On IR targeting of their promoters, presence of secondary siRNAs was excluded for TMM but not for $F T^{12,13}$.

IRs targeting genic regions do not automatically lead to posttranscriptional gene silencing as demonstrated at the FT locus, where IRs targeting the first intron, which contains a repressor binding site, resulted in upregulation of FT expression ${ }^{13}$.

For the transgenic lines containing an IR targeting Block $C$, we mapped IR-induced smRNAs to the whole genome to exclude the presence of off-targets and assess the spreading of smRNAs out of the IR target region. IR-generated smRNAs were found exclusively in transgenic lines and mapped only at the IR target region of the FT locus. Although smRNAs were confined to sequences contained in the IR target region, DNA methylation spread out bidirectionally from the target region up to about $150 \mathrm{bp}$. Previous studies showed that unidirectional spreading of DNA methylation out of the IR target was linked to the generation of secondary siRNAs ${ }^{24,25}$. However, the viral enhancer used as a target in these studies showed an intrinsic promoter activity that may explain the difference in DNA methylation spreading observed with Block $C^{24}$. We conclude that secondary siRNAs are not needed for the limited spreading of IR-mediated DNA methylation in the case of Block $C$.

Although the quantity of smRNAs generated differed by almost one order of magnitude between the transgenic lines, FT downregulation and delay of flowering was similar, indicating a saturated effect. In general, we observed less variation in flowering time between transgenic lines transformed with the same IR construct than with previous promoter-reporter lines for $F T^{1,9,26}$.

The non-transgenic siblings that had lost the IR targeting Block $C$ contained a low but noticeable residual symmetric CG methylation. These plants also showed a mild late-flowering phenotype, which was not always statistically significant across experiments but in most cases apparent. The lack of smRNAs targeting Block $C$ in these plants indicates that METHYLTRANSFERASE 1 (MET1) maintained partial CG methylation across generations. This is in accordance with previous data showing that maintenance of CG methylation was unstable outside siRNA-generating TE regions ${ }^{27,28}$. However, it is unclear whether partial maintenance of CG methylation in the non-transgenic siblings could explain their mild late-flowering phenotype. The fraction of methylated cytosines was so low that Block $C$ is likely to be unmethylated in most cells. Furthermore, the degree of CG methylation maintenance differed between non-transgenic sibling lines, although these showed very similar delays in flowering. On the other hand, if methylation was preferentially retained in FT expressing tissues, it could be causative for the delay in flowering.

Both Block B and Col-0 insertion regions have been shown to interact with the FT promoter in independent reports ${ }^{2,9}$. Targeting of either region with an IR showed a comparable mild effect on flowering time, indicating rather a general chromatin change at the locus than the silencing of a putative cis-regulatory element in these regions. Thus, our data neither support nor contradict an involvement of either region in higher order chromatin structure formation and $F T$ regulation. On the contrary, the stronger lateflowering phenotype of several independent transgenic IR lines for Block $C$ and Block E suggests an implication of these regions in FT regulation. Block $E$ overlaps with a ChIP-seq peak for PIF4 (ref. ${ }^{21}$; Supplementary Fig. 5a) and ChIP-chip peak for SCHLAFMÜTZE (Supplementary Fig. 5b), which is downregulated through the 
age-dependent pathway ${ }^{29}$. Although Block $E$ did not seem required to drive expression in the context of $F T$ promoter-reporter gene constructs $^{1,9,30}$, we show that Block $E$ is sufficient to drive GUS expression in the phloem, as observed previously for Block $C^{1}$. In addition, BlockErev:BlockA::GUS lines show long day induction, indicating a contribution to photoperiod-dependent activity. F1 crosses between Block $E$ and Block $C$ IR transgenic lines are later flowering than their parents, indicating that both enhancers contribute to FT expression in an additive manner. The presence of two enhancers, located upstream and downstream of the gene, may allow a more robust control of FT expression in response to internal or environmental cues. In this context, Block $E$ can be described as a shadow enhancer reported in plants, in the sense that it acts as a separable enhancer driving a similar pattern of expression ${ }^{31}$. The TFBSs responsible for Block $E$ activity and their putative role in regulating FT expression in other tissues and stages remain to be investigated.

In conclusion, FT seems to be regulated by multiple enhancers that seem to have an additive effect on flowering time in inductive conditions. The presence of multiple enhancers seems to be the rule rather than the exception in animals but has not yet been previously demonstrated in plants ${ }^{32-35}$. Enhancer characterization using reporter gene assays may easily overlook such redundant regulatory regions; furthermore, their requirement may be dependent on the genomic context. Here, the FT locus was used as model to show that IR-mediated DNA methylation is an interesting tool to characterize putative cis-regulatory elements showing specific features such as conserved sequence or increased chromatin accessibility in their native chromatin context.

\section{Methods}

Plasmid construction and generation of transgenic plants. To generate IR transgenic lines, defined target regions were PCR amplified from $A$, thaliana Col-0 genomic DNA with oligonucleotides listed in Supplementary Table 1. PCR products were transferred into the Donor vector pDONR207 (Invitrogen) by $\mathrm{BP}$ reaction and then transferred into the destination vector pJawohl8-RNAi (AF408413, http://www.ncbi.nlm.nih.gov/nuccore/AF408413) by LR reaction. Agrobacterium tumefaciens strain GV3101 (pMP90RK) ${ }^{36}$ was used for floral dipping of $A$. thaliana accession Col-0. T1 seedlings at stage four true-leaves were sprayed every four days, four times in total with a solution of glufosinateammonium (250 mgl, BASTA, Bayer). For Block C IR construct, a first visual screen was performed and eight late-flowering lines were kept for selfing and T2 and T3 screen for homozygous lines. Two homozygous and single-copy transgenic lines were obtained (no. 15-2 and no. 27-4). To assess further the potential bias of T1 selection for late flowering, we performed a new visual screen T1 seedlings and could retrieve two T3 single-copy homozygous lines for late and early flowering, respectively. The four lines were scored for flowering time at generation T3 and all of them were late flowering compared to Col-0 (Supplementary Fig. 9a,b).

To generate GUS reporter lines, the destination vector plasmid pGreen0229 was used ${ }^{1}$ and Block $E$ was cloned using Gateway system in sense and antisense directions. For the GUS reporter lines, number of T-DNA insertion copies was estimated by qPCR in T1 plants and only $\mathrm{T} 1$ lines with one copy were kept for GUS staining. Briefly, T1 seedlings were treated with glufosinate-ammonium as described for IR constructs. Genomic DNA was extracted from leaf tissue of at least 15 glufosinate-resistant T1 plants using the BioSprint 96 DNA Plant kit (Qiagen, Catalogue No. 941557). A calibration curve with a serial dilution was made with the home-made plasmid pBS-PP2A-BAR containing one copy of the $P P 2 A$ gene and one copy of the bar resistance cassette (see map in Supplementary Information). qPCR on LightCycler480 (Roche) was performed on a $10 \mu \mathrm{l}$ PCR mix $(0.5 \mu \mathrm{l} 20 \times$ EvaGreen dye (Biotium, Catalogue No. 31000$), 2 \mu \mathrm{l}$ of template, $0.25 \mu \mathrm{l}$ forward primer and reverse primer $(0.25 \mu \mathrm{M}$ each), $0.1 \mu \mathrm{l}$ Taq polymerase (BioBudget, Catalogue No. 80-60010100), $1 \mu \mathrm{l}$ of $10 \times$ buffer $(0.7 \mathrm{mM}$ dNTPs, $0.14 \mathrm{M}$ Tris- $\mathrm{HCl} \mathrm{pH} \mathrm{8.8,} 0.6 \mathrm{M} \mathrm{KCl}$ and $\left.36 \mathrm{mM} \mathrm{MgCl}_{2}\right), 5.9 \mu \mathrm{dH}_{2} \mathrm{O}$ ). The amplification cycle was $95^{\circ} \mathrm{C} 10 \mathrm{~s}, 60^{\circ} \mathrm{C} 15 \mathrm{~s}, 72^{\circ} \mathrm{C} 20 \mathrm{~s}$ ( 40 cycles), followed by a melting curve analysis of $95^{\circ} \mathrm{C} 5 \mathrm{~s}, 65^{\circ} \mathrm{C} 1 \mathrm{~min}$, increase from 65 to $97^{\circ} \mathrm{C}$ with $2.5^{\circ} \mathrm{C}$ per s. For each sample, the ratio of the relative DNA concentrations of bar on PP2A provides an estimation of T-DNA insertion copy number ( 0.5 for a single copy). Oligonucleotides are listed in Supplementary Table 1.

Culture conditions. For ChIP, GUS and smRNA sequencing experiments, seeds were grown on solidified Murashige and Skoog basal medium (MS) in growth chambers (Percival Scientific) in long day conditions ( $16 \mathrm{~h}$ light $/ 8 \mathrm{~h}$ dark) or short day conditions $\left(8 \mathrm{~h} \mathrm{light} / 16 \mathrm{~h}\right.$ dark) at $22^{\circ} \mathrm{C}$, light intensity of $70-100 \mu \mathrm{mol} \mathrm{m}^{-2} \mathrm{~s}^{-1}$. For FT expression analysis, seeds were sown on soil, stratified for 3 days at $4^{\circ} \mathrm{C}$ and transferred in greenhouses or growth chambers, Long day conditions at $22^{\circ} \mathrm{C}$. For flowering time analysis, stratified seeds were transferred either in growth chambers or greenhouses at $22^{\circ} \mathrm{C}$. Either long day or mid-day $(12 \mathrm{~h} \mathrm{light} / 12 \mathrm{~h}$ dark) conditions were used.

Gene expression analysis. Aerial parts of two- or three-week-old seedlings were collected at ZT16 for RNA extraction using TRIzol (Thermofisher, Catalogue No. 15596018) and RNeasy (Qiagen, Catalogue No. 74903), respectively. Total RNA $(2.5 \mu \mathrm{g})$ was treated with DNase I (Ambion, Catalogue No. AM1906) and complementary DNA was generated using Superscript II reverse transcriptase and T18 oligonucleotide for priming (Life Technologies, Catalogue No. 18080-044). RTqPCR was performed with the LightCycler480 (Roche). Expression of PP2A was used to standardize the RNA samples (oligonucleotides are listed in Supplementary Table 1). Three biological replicates were performed for FT expression analysis. For GUS quantification, a single experiment was performed and three independent transgenic lines were considered as biological replicates (see data in Supplementary Information)

Bisulfite sequencing. Genomic DNA was extracted from the aerial part of 10-dayold using DNeasy Plant (Qiagen, Catalogue No. 69104). Bisulfite conversion was performed using the Epitect Bisulfite Kit (Qiagen, Catalogue No. 59104). The conversion cycle programme and the conversion efficiency verification on locus AT5G66750 was performed as described in Foerster and Mittelsten Scheid ${ }^{37}$. Regions of interest were amplified using degenerated oligonucleotides designed with the Kismeth webtool $^{38}$, cloned into TOPO vector using the TA cloning kit (ThermoFisher, Catalogue No. K202020). For IR target regions, oligonucleotides were designed to overlap the construct sequence and the flanking regions to avoid amplifying the T-DNA sequence. Colony PCRs were performed on about 10-12 white colonies using M13 oligonucleotides. Purified PCR products were sequenced using M13 reverse oligonucleotide on 3730XL Genetic Analyzer (Applied Biosystems). Sequences were aligned with SeqMan Pro (DNASTAR) and DNA methylation was analysed with CyMATE $^{39}$. Oligonucleotides are listed in Supplementary Table 1.

smRNA sequencing. Total RNA was extracted from two-week-old seedlings using TRIzol (Thermofisher) following the manufacturer's protocol. Quality check of the RNA was performed on the Agilent 2100 Bioanalyzer before and after the smRNA purification using PureLink miRNA Isolation Kit (Ambion) following the manufacturer's protocol. Libraries for the Block $C$ and Block E IR lines were prepared with the NEBNext Multiplex Small RNA Library Prep Set for Illumina (NEB, Catalogue No. E7300L) and the NEXTflex Small RNA-Seq Kit v.3 (Bioo Scientific, Catalogue No. NOVA-5132-05), respectively. Block C IR and Block E IR libraries were sequenced on Illumina HiSeq 2500 mode 100-bp single reads and Illumina HiSeq 3000 mode 150-bp single reads, respectively. Seven to ten million reads were generated per library. The adaptors were trimmed using Cutadapt ${ }^{40}$, and the reads were mapped on the Arabidopsis TAIR10 reference genome using BWA$\mathrm{ALN}^{41}$. Some residual nucleotides from the adaptors on the reads of the Block $C$ IR libraries were removed with an in-house script and the plots of read coverage were created using $\mathrm{R}^{42}$ (see scripts in https://github.com/johanzi/scripts_Zicola_2019).

ChIP. ChIP was performed as previously described ${ }^{43}$ using two-week-old seedlings harvested at ZT16. A volume of $1 \mu \mathrm{l}$ of rabbit pre-immune serum (Eurogentec, DE10103, rabbit SA66993, PPI2485) was used as no-antibody control and $4 \mu \mathrm{l}$ of H3K9me2 antibodies (Diagenode pAb-060-050, lot no. A90-0042) were used for immunoprecipitation. qPCR was performed with the LightCycler480 (Roche). Oligonucleotides used for qPCR quantification listed in Supplementary Table 1.

Statistical analysis. Statistical analyses were performed with R v.3.5.0 (ref. ${ }^{42}$ ). Homoscedasticity and normality of residuals distribution was tested before either an ANOVA followed by a Dunnett's post hoc test. As non-parametric test, the Kruskal-Wallis multiple-comparison test was used (alpha risk $=5 \%$ ). For flowering time and gene expression analyses, generations were analysed separately (see scripts and data in https://github.com/johanzi/scripts_Zicola_2019).

GUS histochemical staining. Two- or three-week-old whole seedlings were stained for histochemical GUS detection as described previously ${ }^{1}$. Stained samples were mounted on microscopic slides in $50 \%$ glycerol and imaged using the stereomicroscope SMZ18 (Nikon).

Genome comparison. Alignments of the FT locus across Brassicacea species and phylogenetic shadowing at Block $E$ were performed using the VISTA-point tool ${ }^{44}$ (see the method in https://github.com/johanzi/phylogenetic_shadowing).

Reporting Summary. Further information on research design is available in the Nature Research Reporting Summary linked to this article.

\section{Data availability}

All data and materials generated in this study are available without restriction. Sequencing data for smRNA-seq are available in NCBI (BioProject PRJNA427142). Scripts and additional data are available on the GitHub repository https://github. com/johanzi/scripts_Zicola_2019. 
Received: 2 January 2018; Accepted: 25 January 2019;

Published online: 04 March 2019

\section{References}

1. Adrian, J. et al. Cis-regulatory elements and chromatin state coordinately control temporal and spatial expression of FLOWERING LOCUS T in Arabidopsis. Plant Cell 22, 1425-1440 (2010).

2. Cao, $\mathrm{S}$. et al. A distal CCAAT/NUCLEAR FACTOR Y complex promotes chromatin looping at the FLOWERING LOCUS T promoter and regulates the timing of flowering in Arabidopsis. Plant Cell 26, 1009-1017 (2014).

3. Gnesutta, N. et al. CONSTANS imparts DNA sequence-specificity to the histone-fold NF-YB/NF-YC dimer. Plant Cell 29, 1516-1532 (2017).

4. Suárez-López, P. et al. Constans mediates between the circadian clock and the control of flowering in Arabidopsis. Nature 410, 1116-1120 (2001).

5. Ben-Naim, O. et al. The CCAAT binding factor can mediate interactions between constans-like proteins and DNA. Plant J. 46, 462-476 (2006).

6. Wenkel, S. et al. Constans and the ccaat box binding complex share a functionally important domain and interact to regulate flowering of Arabidopsis. Plant Cell 18, 2971-2984 (2006)

7. Shlyueva, D., Stampfel, G. \& Stark, A. Transcriptional enhancers: from properties to genome-wide predictions. Nat. Rev. Genet. 15, 272-286 (2014).

8. Weber, B., Zicola, J., Oka, R. \& Stam, M. Plant enhancers: a call for discovery. Trends. Plant. Sci. 21, 974-987 (2016).

9. Liu, L. et al. Induced and natural variation of promoter length modulates the photoperiodic response of FLOWERING LOCUS T. Nat. Commun. 5 , 4558 (2014).

10. Matzke, M. A. \& Mosher, R. A. RNA-directed DNA methylation: an epigenetic pathway of increasing complexity. Nat. Rev. Genet. 15, 394 (2014).

11. Mette, M. F., Aufsatz, W., van der Winden, J., Matzke, M. A. \& Matzke, A. J. Transcriptional silencing and promoter methylation triggered by doublestranded RNA. EMBO J. 19, 5194-5201 (2000).

12. Deng, S. et al. Transcriptional silencing of Arabidopsis endogenes by single-stranded RNAs targeting the promoter region. Plant Cell Physiol. 55, 823-833 (2014).

13. Deng S. \& Chua, N.-H. Inverted-repeat RNAs targeting FT intronic regions promote FT expression in Arabidopsis. Plant Cell Physiol. 56, 1667-1678 (2015).

14. Hamilton, A., Voinnet, O., Chappell, L. \& Baulcombe, D. Two classes of short interfering RNA in RNA silencing. EMBO J. 21, 4671-4679 (2002).

15. Melnyk, C. W., Molnar, A., Bassett, A. \& Baulcombe, D. C. Mobile 24 nt small RNAs direct transcriptional gene silencing in the root meristems of Arabidopsis thaliana. Curr. Biol. 21, 1678-1683 (2011).

16. Lister, R. et al. Highly integrated single-base resolution maps of the epigenome in Arabidopsis. Cell 133, 523-536 (2008).

17. Du, J. et al. Mechanism of DNA methylation-directed histone methylation by KRYPTONITE. Mol. Cell 55, 495-504 (2014).

18. Du, J., Johnson, L. M., Jacobsen, S. E. \& Patel, D. J. DNA methylation pathways and their crosstalk with histone methylation. Nat. Rev. Mol. Cell Biol. 16, 519-532 (2015)

19. Goodstein, D. M. et al. Phytozome: a comparative platform for green plant genomics. Nucleic Acids Res. 40, D1178-D1186 (2012).

20. Zhang, W., Zhang, T., Wu, Y. \& Jiang, J. Genome-wide identification of regulatory DNA elements and protein-binding footprints using signatures of open chromatin in Arabidopsis. Plant Cell 24, 2719-2731 (2012).

21. Pedmale, U. V. et al. Cryptochromes interact directly with PIFs to control plant growth in limiting blue light. Cell 164, 233-245 (2016).

22. Vaistij, F. E., Jones, L. \& Baulcombe, D. C. Spreading of RNA targeting and DNA methylation in RNA silencing requires transcription of the target gene and a putative RNA-dependent RNA polymerase. Plant Cell 14, 857-867 (2002).

23. Eamens, A., Vaistij, F. E. \& Jones, L. Nrpdla and nrpd1b are required to maintain post-transcriptional RNA silencing and RNA-directed DNA methylation in Arabidopsis. Plant J. 55, 596-606 (2008).

24. Kanno, T. et al. A structural-maintenance-of-chromosomes hinge domaincontaining protein is required for RNA-directed DNA methylation. Nat. Genet. 40, 670-675 (2008)

25. Daxinger, L. et al. A stepwise pathway for biogenesis of 24-nt secondary siRNAs and spreading of DNA methylation. EMBO J. 28, 48-57 (2009).

26. Farrona, S. et al. Tissue-specific expression of FLOWERING LOCUS T in Arabidopsis is maintained independently of polycomb group protein repression. Plant Cell 23, 3204-3214 (2011).
27. Vaughn, M. W. et al. Epigenetic natural variation in Arabidopsis thaliana. PLoS Biol. 5, e174 (2007).

28. Becker, C. et al. Spontaneous epigenetic variation in the Arabidopsis thaliana methylome. Nature 480, 245-249 (2011).

29. Mathieu, J., Yant, L. J., Mürdter, F., Küttner, F. \& Schmid, M. Repression of flowering by the miR172 Target SMZ. PLoS Biol. 7, e1000148 (2009).

30. Takada, S. \& Goto, K. Terminal flower2, an Arabidopsis homolog of heterochromatin protein 1, counteracts the activation of FLOWERING LOCUS T by constans in the vascular tissues of leaves to regulate flowering time. Plant Cell 15, 2856-2865 (2003).

31. Barolo, S. Shadow enhancers: Frequently asked questions about distributed cis-regulatory information and enhancer redundancy. BioEssays News Rev. Mol. Cell. Dev. Biol. 34, 135-141 (2012).

32. Lam, D. D. et al. Partially redundant enhancers cooperatively maintain mammalian pomc expression above a critical functional threshold. PLoS Genet. 11, e1004935 (2015).

33. Ruiz-Narváez, E. A. Redundant enhancers and causal variants in the TCF7L2 gene. Eur. J. Hum. Genet. 22, 1243-1246 (2014).

34. Hu, G., Codina, M. \& Fisher, S. Multiple enhancers associated with ACAN suggest highly redundant transcriptional regulation in cartilage. Matrix Biol. J. Int. Soc. Matrix Biol. 31, 328-337 (2012).

35. Degenhardt, K. R. et al. Distinct enhancers at the Pax3 locus can function redundantly to regulate neural tube and neural crest expression. Dev. Biol. 339, 519-527 (2010)

36. Hellens, R. P., Edwards, E. A., Leyland, N. R., Bean, S. \& Mullineaux, P. M. pGreen: a versatile and flexible binary $\mathrm{Ti}$ vector for Agrobacterium-mediated plant transformation. Plant Mol. Biol. 42, 819-832 (2000).

37. Kovalchuk, I. \& Zemp, F. J. Plant Epigenetics: Methods and Protocols (Humana Press, New York, 2010).

38. Gruntman, E. et al. Kismeth: analyzer of plant methylation states through bisulfite sequencing. BMC Bioinformatics 9, 371 (2008).

39. Hetzl, J., Foerster, A. M., Raidl, G. \& Scheid, O. M. CyMATE: a new tool for methylation analysis of plant genomic DNA after bisulphite sequencing. Plant J. 51, 526-536 (2007).

40. Martin, M. Cutadapt removes adapter sequences from high-throughput sequencing reads. EMBnet J. 17, 10-12 (2011).

41. Li, H. \& Durbin, R. Fast and accurate short read alignment with BurrowsWheeler transform. Bioinforma. Oxf. Engl. 25, 1754-1760 (2009).

42. R Core Team. R: A Language and Environment for Statistical Computing (R Foundation for Statistical Computing, 2014); http://www.R-project.org/

43. Reimer, J. J. \& Turck, F. in Plant Epigenetics 631 (eds Kovalchuk, I. \& Zemp, F. J.) 139-160 (Humana Press, New York, 2010).

44. Frazer, K. A., Pachter, L., Poliakov, A., Rubin, E. M. \& Dubchak, I. Vista: computational tools for comparative genomics. Nucleic Acids Res. 32, W273-W279 (2004).

\section{Acknowledgements}

This work was supported by the Marie Curie ITN grant (no. GA-316965), the Deutsche Forschungsgemeinschaft (no. DFG TU-126/6) and core funding of the Max Planck Society.

\section{Author contributions}

F.T., J.Z. and L.L. designed the experiments. J.Z., P.T. and L.L. performed the experiments. J.Z. and F.T. wrote the manuscript. F.T. obtained the funding.

\section{Competing interests}

The authors declare no competing interests.

\section{Additional information}

Supplementary information is available for this paper at https://doi.org/10.1038/ s41477-019-0375-2.

Reprints and permissions information is available at www.nature.com/reprints. Correspondence and requests for materials should be addressed to F.T.

Publisher's note: Springer Nature remains neutral with regard to jurisdictional claims in published maps and institutional affiliations.

(c) The Author(s), under exclusive licence to Springer Nature Limited 2019 


\section{Reporting Summary}

Nature Research wishes to improve the reproducibility of the work that we publish. This form provides structure for consistency and transparency in reporting. For further information on Nature Research policies, see Authors \& Referees and the Editorial Policy Checklist.

\section{Statistics}

For all statistical analyses, confirm that the following items are present in the figure legend, table legend, main text, or Methods section.

$\mathrm{n} / \mathrm{a}$ | Confirmed

$\square$ \. $\square$ The exact sample size $(n)$ for each experimental group/condition, given as a discrete number and unit of measurement

$\square$ \ A statement on whether measurements were taken from distinct samples or whether the same sample was measured repeatedly

$\square$ The statistical test(s) used AND whether they are one- or two-sided

$\square$ Only common tests should be described solely by name; describe more complex techniques in the Methods section.

$\bigotimes \square$ A description of all covariates tested

$\square$ \ A description of any assumptions or corrections, such as tests of normality and adjustment for multiple comparisons

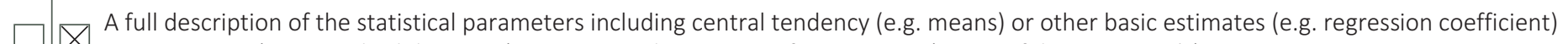

$\square$ AND variation (e.g. standard deviation) or associated estimates of uncertainty (e.g. confidence intervals)

$\square$ For null hypothesis testing, the test statistic (e.g. $F, t, r$ ) with confidence intervals, effect sizes, degrees of freedom and $P$ value noted

$\square$ Give P values as exact values whenever suitable.

Х $\square$ For Bayesian analysis, information on the choice of priors and Markov chain Monte Carlo settings

Х $\square$ For hierarchical and complex designs, identification of the appropriate level for tests and full reporting of outcomes

Х $\square$ Estimates of effect sizes (e.g. Cohen's $d$, Pearson's $r$ ), indicating how they were calculated

Our web collection on statistics for biologists contains articles on many of the points above.

\section{Software and code}

Policy information about availability of computer code

Data collection Roche LightCycler480 software was use to collect qPCR raw data. Illumina BaseSpace analysis was used by the Max Planck Genome Center in Cologne to obtain raw NGS data per barcoded library from an Illumina Hiseq3000 apparatus.

Data analysis

We have added a supplementary information (SI) file containing Extended methods. All custom code that was used to analyze the data
has been submitted to a dedicated github repository (https://github.com/johanzi/scripts_Zicola_2019). The Extended methods sections
contains detailed links to these custom R (v3.5.0) scripts that were used for statistical analyses, plotting of data and NGS-analyses of
smRNA-seq data. The latter script embeds the use of open source software tools: bwa (v0.7.15), bedtools (v2.25.0), R (v3.5.0), fastq-
dump (v2.9.2). We used open source tools webtools to perform sequence alignments (VISTA-Point, no version indicated), Bi-sulfite
conversion (CyMATE, no version indicated) and primer design (Kismeth, no version indicated).
The SI file also contains supplemental sequences that were used for alignments, and raw data tables except if
these are a) deposited in gene bank (smRNA-seq) or b) included in the supplemental data file (raw leaf counts of flowering time assays).
Note that all raw data are redundantly deposited in github.

For manuscripts utilizing custom algorithms or software that are central to the research but not yet described in published literature, software must be made available to editors/reviewers. We strongly encourage code deposition in a community repository (e.g. GitHub). See the Nature Research guidelines for submitting code \& software for further information. 
Policy information about availability of data

All manuscripts must include a data availability statement. This statement should provide the following information, where applicable:

- Accession codes, unique identifiers, or web links for publicly available datasets

- A list of figures that have associated raw data

- A description of any restrictions on data availability

All raw NGS data have been submitted to NCBI as BioProject PRJNA427142. A list of figures with associated raw data tables has been appended to the manuscript text. There are no restrictions to data availability.

\section{Field-specific reporting}

Please select the one below that is the best fit for your research. If you are not sure, read the appropriate sections before making your selection. $\bigotimes$ Life sciences $\quad \square$ Behavioural \& social sciences $\square$ Ecological, evolutionary \& environmental sciences

For a reference copy of the document with all sections, see nature.com/documents/nr-reporting-summary-flat.pdf

\section{Life sciences study design}

All studies must disclose on these points even when the disclosure is negative.

Sample size

All samples were tested post-hoc for normal distribution to select the appropriate statistical test as indicated in the Figure legends and extended methods section. In general, a sample number of 8-12 was considered as sufficient for testing flowering time on defined plant genotypes based on empirical experience. A minimum of three biological replicates was considered as required for replication of gene expression measurements, two independent transgenic lines testing for a common effect were included in all analyses.

Data exclusions For Fig.1b, data for 1 genotype was not shown due to poor germination as indicated in the figure legend (NA). For Fig.1e, non-transgenic segregants of line \#27-3 generation T5 were excluded from the statistical analysis of FT expression differences due to an extreme outlier biological replicate $(n=3)$.

Replication

Flowering time data were replicated either by repeating the entire experiment independently or through the inclusion of a sufficient number of biological replicates as indicated in the corresponding figure legends and methods sections. All results were robustly replicated without exception. Measurements of gene expression by qRT-PCR, smRNA-seq were replicated using independent biological replicates grown and processed in parallel or grown and processed independently as indicated in the corresponding figure legends and methods sections $(n=3)$. The results were robustly replicated with the exception of the data shown in Fig. 1e, for whihc one genotype in one generation was excluded from the statistical analysis due to an extreme outlier data point. DNA-methylation analysis by BS-conversion was performed once on a pool of plants per genotype, 7-12 clones per genotype were analysed for C-T conversion as indicated in figure legends. Two independent transgenic events were tested for effect and showed the same result. ChIP-qPCR was performed on two biological replicates, two independent transgenic events were tested for effect and showed the same result.

Randomization The samples were compared by genotype or genotype/generation. The samples were randomized for location within each experiment to avoid position effects in the greenhouse. Since greenhouse and growth cabinet conditions slightly differ throughout the year, due to external light or artificial light source fluctuations, samples were compared if grown at the same time whenever possible using an adequate number of biological replicates per genotype. An exception are data presented in Extended Figure 1, which contains pooled data from four independent experiments performed in $16 \mathrm{~h} / 8 \mathrm{~h}$ long day conditions. The data corroborate results shown in Figure 1, which represent a single $12 \mathrm{~h} / 12 \mathrm{~h}$ long day experiment.

Blinding $\quad$ Plants used for leaf counts were randomized across the growth space and counted in the order of their location without attention to the genotype. They were, however, clearly labeled at the time of scoring.

\section{Reporting for specific materials, systems and methods}

We require information from authors about some types of materials, experimental systems and methods used in many studies. Here, indicate whether each material, system or method listed is relevant to your study. If you are not sure if a list item applies to your research, read the appropriate section before selecting a response. 


\begin{tabular}{l|l}
\hline$n / a$ & Involved in the study \\
$\square$ & $\bigotimes$ Antibodies \\
$\searrow$ & $\square$ Eukaryotic cell lines \\
$\searrow$ & $\square$ Palaeontology \\
$\searrow$ & $\square$ Animals and other organisms \\
$\searrow$ & $\square$ Clinical data
\end{tabular}

\section{Antibodies}

\section{Antibodies used}

Validation
H3K9me2 antibodies ( $4 \mu \mathrm{l}$ per ChIP, Diagenode pAb-060-050, lot NA90-0042) were used for ChIP-PCR. In house pre-immune ( $1 \mu \mathrm{l}$ per ChIP) serum from rabbit was used as control and is available upon request.

See Diagenode's datasheet for validation of this purified poly-clonal antibody ( https://www.diagenode.com/files/products/ antibodies/Datasheet_H3K9me2_C15410060.pdf) Validation includes ChIP-PCR, ELISA and peptide binding arrays and immunofluorescence for positive samples and a large collection of negative controls. 